\title{
Landscape Ecology
}

Christensen, Andreas Aagaard; Brandt, Jesper; Svenningsen, Stig Roar

Published in:

The International Encyclopedia of Geography

DOI:

10.1002/9781118786352.wbieg1168

Publication date:

2017

Document version

Version created as part of publication process; publisher's layout; not normally made publicly available

Citation for published version (APA):

Christensen, A. A., Brandt, J., \& Svenningsen, S. R. (2017). Landscape Ecology. In D. Richardson, N. Castree, M. F. Goodchild, A. Kobayashi, W. Liu, \& R. A. Marston (Eds.), The International Encyclopedia of Geography: People, the Earth, Environment, and Technology (pp. 1-10). Wiley. Major Reference Works https://doi.org/10.1002/9781118786352.wbieg1168 


\section{Landscape ecology}

\section{Andreas Aagaard Christensen Copenhagen University, Denmark}

Jesper Brandt

Roskilde University Denmark

Stig Roar Svenningsen

The Royal Library, Denmark

The foundations of landscape ecology

The term landscape ecology (in the original German Landschaftsökologie) was first used in 1939 by the German geographer Carl Troll as the name for a particular way of looking upon the landscape (Anschaunngsweise) based on a holistic perspective integrating geographical and ecological insights into the nature of terrestrial environments (Troll 1939). Troll's previous research into the coupled relationship between vegetation, environment, and land use had brought him to conduct extensive fieldwork registrations of vegetation patterns in landscapes in Northern Europe. These experiences, in turn, had inspired him to take up aerial photography as an instrument to identify and describe spatial units of vegetation cover, the heterogeneous pattern of which he was then able to relate analytically to both social and environmental processes of change. On these foundations, Troll proposed a science of landscape processes that would be based on the new technology of aerial photograph interpretation, to classify the earth's surface into discrete land units. On the basis of such inventory classifications, it was possible for Troll and his contemporaries to integrate analytical perspectives from a range of otherwise discrete sciences in their analysis of landscape processes. In this way, the development of landscape ecology, characterized by its specific focus on the ecological significance of spatial form and pattern, was closely associated both with the novel perspective provided by aerial photography and also with older geographical perspectives of analysis inherited from classical cultural and physical geography.

Troll had been trained in the traditions of geography defined by Alexander von Humboldt and Carl Ritter, the two great initiators of the field as a modern science in the middle of the nineteenth century. From them and their immediate successors he had picked up a certain breadth of scope and an interest in the interconnected nature of geographic phenomena, which he emphasized in his writings. He had studied the work of Humboldt and Ritter extensively and had been impressed especially by their ability to correlate natural and cultural phenomena. From Humboldt's scholarship he had understood the need to abstain from disentangling research objects from their environment, but rather to delve into the multifaceted empirical relationships linking otherwise seemingly disparate elements with larger patterns of distribution and causality. For Troll and his contemporaries, it was never enough to understand the nature of a species or habitat type in its own right - the aim was to explain how species and habitats interact with each other and other facets of their environment, with reference to actual, empirical histories of change.

On this basis Troll was increasingly critical of the growing specialization of academic

The International Encyclopedia of Geography.

Edited by Douglas Richardson, Noel Castree, Michael F. Goodchild, Audrey Kobayashi, Weidong Liu, and Richard A. Marston.

(C) 2017 John Wiley \& Sons, Ltd. Published 2017 by John Wiley \& Sons, Ltd.

DOI: $10.1002 / 9781118786352 . w b i e g 1168$ 


\section{LANDSCAPE ECOLOGY}

geography in his day. Since the late nineteenth century the universalist approaches advocated by Ritter and Humboldt had been superseded in mainstream scholarship by an outspoken (and in Troll's view unwarranted) reductionist stance towards the organization of scientific knowledge. Researchers within geography had sought to establish biological, physical, and demographic modes of analysis as distinct fields of research, and within these fields it had become the norm to seek for the smallest possible and most basic units of analysis in order to be able to study each environmental subprocess individually, as if in a laboratory. Troll saw this development as an understandable but tragic detour from the unified perspectives of earlier geography, and one that made it increasingly difficult to correctly observe and describe the cohesive, interdependent nature of geographic phenomena. Therefore, Troll sought to conserve and reformulate a unified perspective on terrestrial surface processes. Hence, landscape ecology from the outset was conceived as an approach to environmental research that was to be unrestrained by disciplinary and methodological dogma.

When, in 1959, Troll was asked to address the geographers of the British Association for the Advancement of Science on the centenary of the death of Humboldt and Ritter, he made his views on contemporary geography remarkably clear. In his speech he located the inspiration for a range of seminal landscape ecological concepts and instruments within the work of his two academic idols. These included: forms of spatial pattern analysis, which Troll became inspired to apply and further develop upon reading Humboldt's pioneering work on ecological zones and associated patterns of plant distribution; the method of regionalization developed by Ritter, which Troll employed to classify heterogeneous areas (i.e., landscapes) into analytical units based on similar form, genesis or character; and types of early socioecological analysis developed by Ritter through his work on the relationship between land use, culture, and civilization (Troll 1960).

These concepts and instruments came to inspire the development of landscape ecology, as a counterbalance to the fragmentation or disintegration of geographic-ecological thought in the twentieth century that motivated landscape ecologists to establish their field as an explicitly integrative science. In the 1950s and 1960s, landscape ecology consisted of a synthesis of geography with soil science and ecology. From the late 1960s especially, island biogeography increased in importance (MacArthur and Wilson 1967). Parallel to this, a corresponding, spatially-oriented vegetation science developed, and among conservation-oriented zoologists a strong school of dispersal ecology and metapopulation theory developed (Gilpin and Hanski 1991). The methods and perspectives of these fields were combined by applying them to the same spatially defined land units at various scales - from single landscape elements or land units to larger complexes of functionally and historically associated sets of elements, up to whole landscapes defined by heterogeneous patterns of landscape elements.

Figure 1 illustrates the type of method for spatial analysis that is typically used. It allows researchers to organize the many mono-disciplinary insights flowing into landscape ecology into a common empirical understanding of landscape dynamics, by referencing all relevant explanatory understandings to a common set of land units and the landscapes they constitute. In this way it becomes possible to examine the spatial distribution and overlap of explanatory understandings spatially based on map analysis. As the field developed, this basic method of integrating different perspectives by way of a common spatial framework remained unchanged, even though the range 

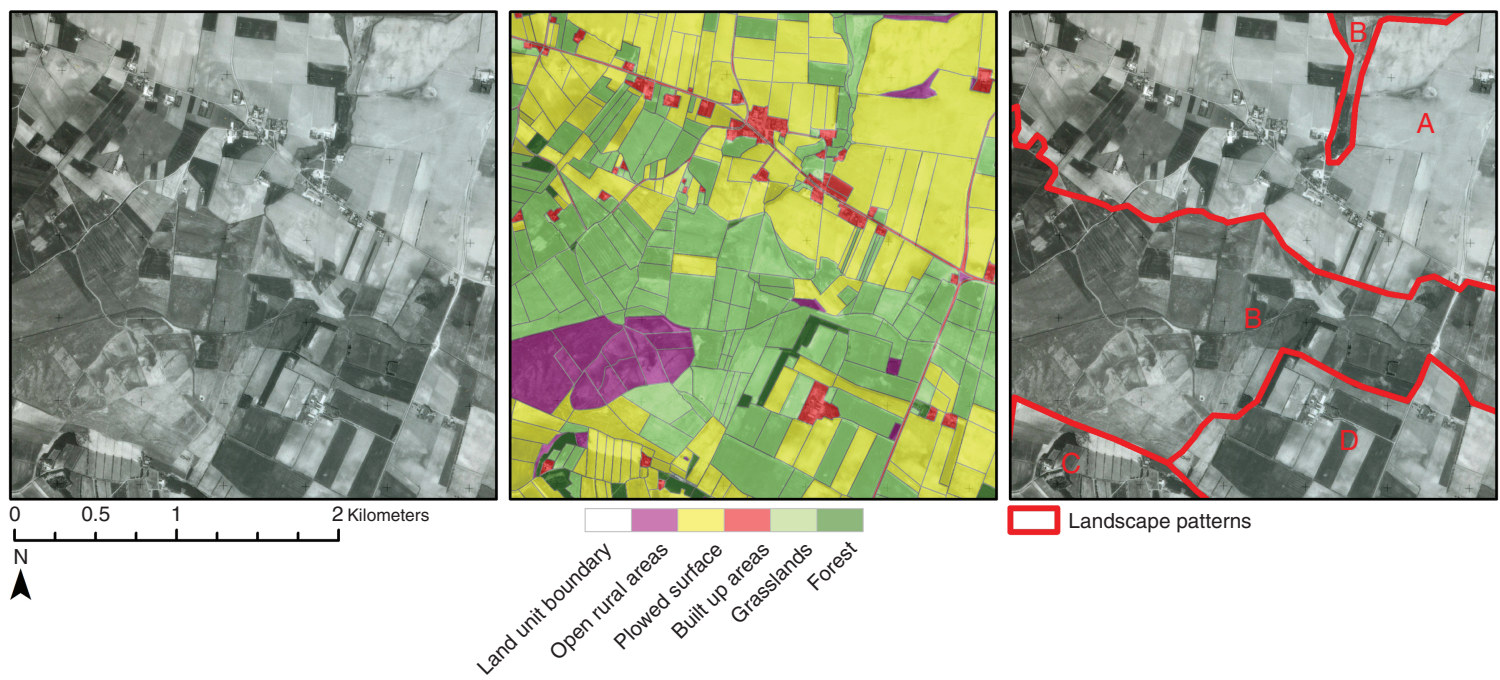

Figure 1 Landscape patterns analysis typically starts with a mosaic of vertical aerial imagery such as the sample shown here (left). Through imagery analysis supported by fieldwork observations, the remote sensed data are transformed into a classified map of internally homogeneous land cover units (center). Considered as a whole, the land cover units form a set of heterogeneous landscape patterns (right), each indicating a particular processual relationship between life forms on the earth surface (including humans) and their geoecological basis. The sample shown here illustrates how rural land use is adapted to the geoecological conditions in an agricultural landscape near Skive in Northern Jutland, Denmark. In the north there is an intensively used and closely settled agricultural landscape dominated by arable fields situated on well-drained loamy soils (A). A narrow erosion valley covered by grassland habitats intersects the arable land from the north, draining into a broad glacial valley of low-lying waterlogged soils covered by paddocks and remnants of moorland at the center of the area (B). South of the central valley there is a relatively complex pattern of agricultural land use, which is adapted spatially and functionally to the heterogeneous soils of the area, characterized by a variety of gravel, sand, and clay deposits (C \& D). Analytical approaches of this kind, which link spatial pattern with landscape functionality and landscape history, are used within landscape ecology to integrate perspectives from ecology, soil science, cultural geography, sociology, and the humanities into a common, spatially explicit framework of analysis. On this basis, otherwise potentially incongruent theories explaining landscape dynamics can be tested and compared. Source: Imagery recorded by the Royal Airforce in 1980, held by the Royal library of Denmark.

of disciplinary components included within it broadened considerably over time.

When the various adverse effects of modern land use development and associated destructive ecological transformations came under increasing public scrutiny in the 1960s and onwards, many landscape ecologists saw a potential in their field to provide integrated solutions for the development of more sustainable landscape management practices through applied research. One of the major driving forces for this development was the realization that ongoing processes of industrialization in the agricultural sector were leading to widespread habitat fragmentation and loss of ecosystem services in European and postcolonial landscapes. These changes were pioneered in Eastern Europe, where they were associated with centrally-planned 
collectivization of agricultural properties into specialized, large-scale agroindustrial units. In Western Europe and North America, agricultural industrialization developed later, in most cases driven by a combination of government subsidies and competition among producers, so landscape ecology as an applied scientific practice developed more slowly and later here than in Eastern Europe (Ruzicka and Miklos 1990).

\section{Cultural landscapes and holism}

The growing influence of applied perspectives meant that landscape ecologists came into sustained conflict with traditional ideals of scientific practice, leading some researchers to define their field as an explicitly action-oriented, solution-driven practice integrating scientific discovery with direct intervention in landscape management practices. This has remained a widespread perspective within landscape ecology, which now includes a comprehensive array of planning and policy-related fields of research. In addition to these developments, landscape ecological research has also extended in the direction of historical and social disciplines. This has taken place gradually as researchers within the field have cultivated an increasingly integrated relationship with scholarship from the human and social sciences, which was considered critical component to include within landscape ecology to fully appreciate the complex trajectories of change in human-dominated cultural landscapes.

Cultural landscapes have remained a dominant research object throughout the history of landscape ecology, which has progressed from descriptive accounts of human-landscape interaction to more intricate attempts at understanding, conceptualizing, and quantifying the nature of socioecological relationships in a landscape context. Such research demands a highly developed model for integrative research, because human landscape management is a process that mediates both between the ecological and social sides of human existence and between natural and anthropogenic facets of ecosystem functioning. As the German geographer Ernst Neef, one of the founders of socioecological research within the field, expressed it: "The transfer from societal changes to the natural systems is based on the spontaneous effect of the laws of nature, whereas the transfer from the societal area to the regulation of natural processes is achieved by cultural forces, human perception and decision making" (Neef 1984, 6). As such, cultural landscapes are a human construct and a biophysical system at the same instance, and any attempt to analyze, theorize or interfere with landscape dynamics must be able to account for both of these dimensions. Within landscape ecology the challenge of accounting for these two sides of landscapes in an integrated way has motivated the formulation of a landscape concept where the landscape is seen as a holon: an assemblage of interrelated phenomena that together forms a complex whole, which is "more than the sum of its elements" because "all parts are internally related to each other by the general state of the whole" (Naveh 2000, 11).

This perspective has become a characteristic feature of landscape ecological research. It explicitly challenges both the basic division between anthropogenic and natural phenomena, as well as the mono-disciplinary reductionism prevalent in mainstream science, by claiming that the socioecological processes constituting landscape holons and their constituent parts cannot be understood without accounting for the multifaceted relationships that unite individual elements into a spatially and functionally integrated whole. 
In terms of empirical work, such relationships have been investigated by focusing on the processes or "functions" linking ecosystems with each other and with human agency. In this way, empirical evidence of the organization of landscape holons is collected by characterizing and mapping the extent, magnitude and coupled interplay of ecosystems processes (ecosystem functionality), social processes (societal functionality), and intentional practice (transcending functionality) within landscapes (Brandt and Vejre 2004). By integrating intentional human practices related to human culture, cognition, belief, planning, and decision-making with respect to landscapes into the field on equal terms with other more tangible types of functionality, landscape ecologists were able to outline a broad-ranging holistic science devoted to examining the full interplay between socioecological processes and biophysical patterns in landscapes (Nassauer 1997).

\section{Aerial vision: a new perspective in the} study of landscapes

From the outset, landscape ecology was characterized by a visual approach to analyzing landscapes from a birds-eye perspective, making it possible to detect minute nuances in land cover patterns, while at the same time retaining a distanced landscape-scale overview. This perspective was made possible mainly due to the new technology of vertical mono- and stereographic aerial photography, which became gradually more widespread in the period after World War I. The technology of aerial photography had been pioneered with recordings from balloons over Paris in the 1850s and in Boston some years later, but due to navigational challenges the method proved unsuited for systematic recordings of large areas.
It was the advent of airplanes in the early twentieth century that kicked off what was to become an explosion of vertical aerial photography. At first, images were recorded in limited numbers and with rudimentary instruments, but technological development and funding for recording campaigns soon became fueled by public investment as the potentials for military use of the imagery became evident. During World War I recording efforts grew rapidly. Mobile laboratories for photographic processing became available in 1915, making it possible to analyze images swiftly at locations along the front lines. This meant that aerial photography became a critical technology to the war effort, and the increasing entrenchment of the western front raised a demand for intensive small-scale scrutiny of long stretches of frontline, to a point where at the height of the war the French side alone was processing more than 10000 images each night. In the period after World War I, images and recording technology became available for other uses, forming a growing resource for landscape research, and a broad spectrum of researchers, planning, and policy professionals picked up the new technology.

The idea of an all-encompassing birds-eye view of the earth's surface goes back to antiquity and cartographic representations of the earth as seen from above formed part of established practices within the scientific community long before airplanes made it possible to actually see the earth's surface from above. In spite of this, however, it had an unanticipated effect on landscape research when actual remote sensed imagery became available. Earlier map-makers based their delineations of areas, lines, and point features on fieldwork observations collected on the earth's surface, which were then transposed onto a spherical or flat medium in order for patterns of landscape elements to be made available for visual inspection "as if" seen from 


\section{LANDSCAPE ECOLOGY}

above. In this sense, all earlier maps reflect a form of inquiry where research design, sampling, and data collection precedes the rendition of raw data spatially. In such mapping processes, the data becomes spatial in coverage only by way of analysis, not beforehand.

When remote sensed imagery eventually became available, it represented an altogether different type of resource for map-makers than previous types of spatial data. Mosaics of aerial imagery offered researchers a continuous field of spatial data to begin with, making the earth's surface an observable research object in its own right, rather than a canvas on which to drape existing observations. And when series of images of the same areas at different points in time were recorded, it also became possible to classify processes of landscape change by way of direct overlay analysis. This was the key condition for the development of early landscape ecological analysis methods, and for the particular concept of landscape processes that still characterizes the field.

In the period since World War II, the range, coverage, and resolution of remote sensed imagery available for scientific analysis has grown rapidly. The Cold War, which succeeded World War II and culminated with the formal dissolution of the USSR in December 1991, meant that American and Russian intelligence gathering efforts were in constant preparation for armed conflict. New, more precise recording devices and new platforms for carrying them, such as the U2 high altitude jets and the Corona and Landsat satellite programs, are examples of the tremendous development of remote sensing technology that took place. When imagery from these programs was gradually declassified and older platforms began to be replaced by new in a succession of technological improvements, a broad range of new materials were added to the repertoire of data available for landscape ecological analysis. This spurred a rapid development of spatial analysis tools within the field, supported by the new technology of user-centered computing, which became available from the 1970s onwards.

As such, landscape ecology became one of several arenas where geographical information systems (GIS) and associated geostatistical tools were pioneered and developed. In conjunction with these efforts, an array of computational approaches and indexing-methods for measuring and comparing the configuration, complexity, and diversity of land cover patches was developed, including the important Fragstats software package that empowered individual researchers to conduct quantitative spatial analysis (McGarigal and Marks 1995). This initiated a shift in landscape ecology away from analogue pattern recognition towards the use of computational tools able to quantitatively assess landscape patterns and associated processes across vast expanses of land.

\section{Current methods}

Current landscape ecological methods involve a combination of fieldwork and analysis of imagery to identify the relationship between patterns and processes. Pattern in this sense refers to heterogeneity in the horizontal dimension of the landscape, i.e. differences between land units, while ecological processes typically transpire within a single land unit and refer to the vertical relationships between organisms and their abiotic environment. The various approaches within the field relate to either of these two parallel analytical perspectives: (1) the chorologic, dealing with the horizontal patterns and processes of land units on the earth surface, and (2) the topologic, dealing with the vertical processes of energetic, informational and material exchange 
between organisms within their habitats (Neef 1963; Zonneveld 1989).

In a chorologic perspective the primary dimensions of the landscape are its geometrical and temporal characteristics. Here landscape dynamics are approached analytically by measuring alterations in the shape, extent, and distribution or pattern of land units in the landscape, and by analyzing exchanges occurring between individual units. Trajectories of landscape change through time can be appraised by determining the rate of change, its frequency, and magnitude (Antrop 2000).

In a topologic perspective the character of a land unit is investigated by examining its internal functioning as defined by the societies of species within it and its geoecological potentials in the form of substrates, climate, and hydrology, including the flows of information, matter, and energy taking place through vertical vectors within it.

Today, the patch-corridor-matrix model introduced by Richard T.T. Forman and Michael Godron has become the most widespread conceptual model with which to approach pattern-process relationships (Forman and Godron 1986). It consists of a spatial language designed to describe landscape patterns and related processes. The basic idea of the model is that landscapes are made up of a mosaic of patches (areas differing from their surroundings), connected by corridors (strips of land that infiltrate the landscape and support flows of information, matter, and energy) in a matrix (defined as the dominant, most extensive, and coherent landscape element type) (Forman 1995). In this perspective a landscape is defined by the pattern formed by patch, corridor, and matrix elements repeated throughout its extent. By measuring the size, shape, and distribution of these three types of elements, landscapes can be compared quantitatively in a number of ways. Key parameters include the connectivity, diversity, and composition of landscapes, which have been shown to be systematically associated both with types of human land-use practices affecting landscapes, with biodiversity, and with ecosystem functioning within patches.

\section{Landscape ecology today and its} challenges

Landscape ecology has grown to become a widely recognized approach to environmental research, distinguishable from other scientific traditions by the type of spatially explicit, interdisciplinary, and empirically-focused analytical perspectives detailed above. By 1980 researchers from across the world had become involved in the development of the field, with a world conference convened in the Netherlands in 1981. The following year the International Association for Landscape Ecology (IALE) was established as a focal point for dialog and mutual exchange of ideas by organizing regular conferences and through publication of newsletters and journals. These organizational activities take place at international, regional, and national levels through local chapters of the association. They have proven to be instrumental for the further development of landscape ecology, because the local working environment of many landscape ecologists is delimited by traditional mono-disciplinary or sectoral organizational structures and associated domains of expertise within the academic community. Therefore, most landscape ecological research relies on the application of a combination of skills and insights from researchers and practitioners who retain their commitment to one or more disciplines while contributing to landscape ecology through cross-, inter- and transdisciplinary cooperation. On this basis, a substantial literature on how to 
facilitate interdisciplinary exchange and organize action research has developed within landscape ecology, which has come to form a significant scientific contribution in its own right (Tress, Tress, and Fry 2005).

But, at the same time, landscape ecology is also haunted by its fragmented character, and one of the most persistent threats to its continued success is the challenge of translating concepts and methods that have been harvested eclectically from other disciplines into a common analytical framework. These efforts are made increasingly difficult by the fact that the field itself has become subdivided to some degree, because researchers from different parts of the world are basing their analytical efforts on differing theories of science and validity, and thus also on different criteria for scientific achievement within the field. Today, mainstream American and European approaches differ in research priorities and theoretical perspectives for example, which is reflected in books designed to provide an overview of the field from either of the two perspectives. The introductory text by Bastian and Steinhardt is a European example, while the reader compiled and commented by Wiens et al. is an American parallel (Bastian and Steinhardt 2002; Wiens et al. 2007).

In a global perspective, the main differences between perspectives within landscape ecology, including those between American and European scholars, have tended to reflect underlying disparities concerning: (i) the way in which human interference or engagement with landscape processes is handled analytically and theoretically; (ii) the degree to which researchers lean towards epistemologies derived from the human sciences and/or the natural sciences; and (iii) the degree of practical engagement with landscape management and policymaking.

When landscape ecological research is reviewed along these lines it is clear that a particular disparity within the field tends to inform and support the others, namely that between approaches advocating theoretical pluralism on the one hand, and approaches characterized by exclusive theories of truth and validity on the other. This appears to be a division line that cuts across other variations within the field, and it has become particularly clear with respect to research identified either with a strict positivist stance or with a constructivist conception of truth.

This may illustrate that while landscape ecologists have succeeded in reconciling their multifaceted perspectives on the landscape when dealing with tangible empirical problems, policy advice, and action research, it has proven more difficult to resolve differences pertaining to the variety of underlying, a priori theoretical foundations within the field. Landscape ecology was united not by a common theoretical debate but rather by a set of methods and research interests, and a fascination with a common empirical domain. At times this has been a great advantage to landscape ecology, which has been able to include and cultivate a host of different perspectives, but it also makes it a challenge to uphold a unified perspective able to make good on Carl Troll's original ambition to understand landscapes holistically.

This would entail the further parallel development and amalgamation of three existing subject areas within landscape ecology.

1 Research focusing on the basic, persistent structural and processual character of landscape types and processes of landscape change, which provides insights into the long-term natural history of landscapes, enabling society to better understand and adapt to general conditions for land-use management.

2 Studies of historic and actual, anthropogenic and natural landscape structures and their development through time, which illustrate 
past and present relationships and barriers within landscapes, supporting improved understandings of how to conserve and/or improve valuable landscape resources.

3 Studies of the cultural and ideological dimensions of human engagement with landscapes and associated types of management practices, making it possible to understand the sociocultural and political background for sustainable landscape management.

The main challenge for landscape ecology, therefore, is not to focus or broaden its scope, but rather to improve the way in which insights gained in one area of the field are communicated, compared, and combined with insights from other areas.

SEE ALSO: Cultural geography; Landforms and physiography; Landscape

\section{References}

Antrop, Marc. 2000. "Background Concepts for Integrated Landscape Analysis." Agriculture, Ecosystems \& Environment, 77 (1-2): 17-28. DOI: 10.1016/ S0167-8809(99)00089-4.

Bastian, Olaf, and Uta Steinhardt, eds. 2002. Development and Perspectives of Landscape Ecology. Dordrecht: Kluwer Academic.

Brandt, J., and H. Vejre. 2004. "Multifunctional Landscapes - Motives, Concepts and Perspectives." In Multifunctional Landscapes - Theory Values and History, edited by J. Brandt and H. Vejre, 13-31. Ashurst, UK: WIT Press.

Forman, Richard T.T. 1995. Land Mosaics: The Ecology of Landscapes and Regions. Cambridge : Cambridge University Press.

Forman, Richard T.T., and Michel Godron. 1986. Landscape Ecology. New York: John Wiley \& Sons.
Gilpin, M., and I. Hanski, eds. 1991. Metapopulation Dynamics: Empirical and Theoretical Investigations. London: Academic Press.

MacArthur, R. H., and E. O. Wilson. 1967. The Theory of Island Biogeography. Princeton, NJ: Princeton University Press.

McGarigal, K., and B.J. Marks. 1995. Fragstats - Spatial Pattern Analysis Program for Quantifying Landscape Structure. Corvallis: Oregon State University.

Nassauer, Joan Iverson, ed. 1997. Placing Nature: Culture and Landscape Ecology. Washington, DC Island Press.

Naveh, Zev. 2000. "What Is Holistic Landscape Ecology? A Conceptual Introduction." Landscape and Urban Planning, 50(1-3): 7-26. DOI: 10.1016/ S0169-2046(00)00077-3.

Neef, E. 1963. "Topologische Und Chorologische Arbeitsweisen in Der Landschaftsforschung [Topologic and Chorologic Approaches in Landscape Research]." Petermanns Geographische Mitteilungen, 107(4): 249-259.

Neef, E. 1984. "Applied Landscape Research." Paper Dedicated to the Participants of The First International Seminar of The International Association for Landscape Ecology (IALE), Roskilde, Denmark.

Ruzicka, M., and L. Miklos. 1990. "Basic Premises and Methods in Landscape Ecological Planning and Optimization." In Changing Landscapes. An Ecological Perspective., edited by Isaak S. Zonneveld and Richard T.T. Forman. New York: Springer Verlag. Tress, Gunther, Bärbel Tress, and Gary Fry. 2005. "Clarifying Integrative Research Concepts in Landscape Ecology." Landscape Ecology, 20(4): 479-493. DOI: 10.1007/s10980-004-3290-4.

Troll, Carl. 1939. "Luftbildplan Und ökologische Bodenforschung : Ihr Zweckmäßiger Einsatz Für Die Wissenschaftliche Erforschung Und Praktische Erschließung Wenig Bekannter Länder [Aerial Imagery and Ecological Earth Science]." Zeitschrift der Gesellschaft für Erdkunde zu Berlin, 7: 241-298.

Troll, Carl. 1960. "The Work of Alexander von Humboldt and Carl Ritter. A Centenary Address." The Advancement of Science, 16(64): 441-452. 


\section{LANDSCAPE ECOLOGY}

Wiens, J.A., M.R. Moss, M.G. Turner, and D.J. Mladenoff, eds. 2007. Foundation Papers in Landscape Ecology. New York: Columbia University Press.

Zonneveld, Isaak S. 1989. "The Land Unit - A Fundamental Concept in Landscape Ecology, and Its Applications." Landscape Ecology, 3(2): 67-86. DOI: 10.1007/BF00131171.

\section{Further reading}

Forman, R.T.T., and M. Godron. 1986. Landscape Ecology. New York: John Wiley \& Sons.
Leser, H. 1976. Landschaftsökologie [Landscape Ecology]. Stuttgart, Germany: Eugen Ulmer.

Naveh, Z., and A. Lieberman. 1984. Landscape Ecology. Berlin: Springer Verlag.

Zonneveld, Isaak S., and Richard T.T. Forman, eds. 1990. Changing Landscapes: An Ecological Perspective. New York: Springer. 
Please note that the abstract and keywords will not be included in the printed book, but are required for the online presentation of this book which will be published on Wiley Online Library (http://onlinelibrary.wiley.com/). If the abstract and keywords are not present below, please take this opportunity to add them now.

The abstract should be a short paragraph of between 150-200 words in length and there should be 5 to 10 keywords

\begin{abstract}
Landscape ecology is an interdisciplinary field of research and practice that deals with the mutual association between the spatial configuration and ecological functioning of landscapes, exploring and describing processes involved in the differentiation of spaces within landscapes, and the ecological significance of the patterns which are generated by such processes. In landscape ecology, perspectives drawn from existing academic disciplines are integrated based on a common, spatially explicit mode of analysis developed from classical holistic geography, emphasizing spatial and landscape pattern analysis and ecological interaction of land units and using aerial photography. The landscape is seen as a holon: an assemblage of interrelated phenomena, both cultural and biophysical, that together form a complex whole. Enduring challenges to landscape ecology include the need to develop a systematic approach able to translate positivist readings of the environment and hermeneutical perspectives on socioecological interaction into a common framework or terminology.
\end{abstract}

Keywords: aerial photography; ecology; ecosystems; cultural geography; cultural landscapes; holon; landscape; regionalization; remote sensing; socioecological analysis; spatial pattern analysis 